\title{
POST MI PERICARDIAL EFFUSION-“SIZE DOES MATTER"
}

\section{G.Abishek Ravi Varma}

\begin{abstract}
:
Post myocardial infarction pericardial effusion (PE) is a relatively common, however the prognosis and management is not always straightforward in patients with moderate pericardial effusion (MPE). This article describes four case scenarios we encountered and reviews existing literature regarding etiology and management of post MI pericardial effusion.
\end{abstract}

Key words: Pericardial effusion, Management, Post MI.

\section{INTRODUCTION}

Pericardial effusion (PE) associated with myocardial infarction (MI) is considered to be associated with anterior ST-segment elevation MI (STEMI), with large infarcts, and when heart failure is present. When PE is associated with cardiac tamponade, it is usually due to cardiac rupture, hemorrhagic pericarditis, or aortic dissection involving the right coronary artery [1]. However, the majority of PE does not cause hemodynamic compromise, and a small to moderate PE may often be viewed as a benign reaction to the MI. In a large study in the modern era of reperfusion from 25 French hospitals including 908 patients with MI, a PE was detected in only $6.6 \%$ of the patients [2].Patients had an echocardiogram performed at admission and again at discharge, and $0.8 \%$ developed left ventricular free wall rupture (FWR). This incidence of FWR is similar to that of a larger registry that did not search meticulously for rupture [3].

Acute FWR is one of the most common causes of mortality in acute MI. The diagnosis is most often made by the presence of sudden electromechanical dissociation followed by death if left untreated. Some patients survive for several hours, allowing time for

Article received on 01 OCT 2016, published on 31 OCT 2016.

G.Abishek Ravi Varma ${ }^{1}$,

${ }^{1}$ DM student, Department of Cardiology, NIMS, India

Corresponding author: G. Abishek Ravi Varma

Email: abishekcrazy@gmail.com diagnosis and intervention. Large systematic series have shown that rupture is most common on day 1 [4]. We describe 4 cases in the next section about various presentation of post MI moderate pericardial effusion.

\section{CASE REPORTS}

\section{Patient 1}

A 58 year old hypertensive female, on regular medications, presented with cardiogenic shock, following a five day history of chest pain. At admission her Electrocardiography (ECG) revealed an infero-lateral myocardial infarction (MI), and 2D echo showed RWMA in infero posterior wall, mild LV dysfunction and moderate pericardial effusion with obvious signs of tamponade, immediate pericardiocentesis via subxiphoid approach was performed with a pigtail catheter placed in situ , $400 \mathrm{ml}$ of haemorraghic fluid was drained following which her condition improved dramatically. However the following day the patient complained of persistent chest discomfort and her BP was showing a deceasing trend. ECG revealed recurrent ST segment elevations, repeat 2D echo showed pericardial effusion with signs of tamponade and pericardiocentesis was performed through the pigtail previously placed, $350 \mathrm{ml}$ was drained subsequently she became stable. At this juncture the possibility of free wall rupture was considered in view her persistent pain, recurrent pericardial effusions and hypotension. Over the next few days she was managed conservatively and discharged with plan to perform CAG at a later date.

On $2^{\text {nd }}$ admission patient was stable and 2D echo revealed no further pericardial effusion, CAG was performed which showed DVD (LCX $90 \%$ and RCA 90 $\%$ stenosis) subsequently PTCA was performed.

\section{LEARNING POINT}

1) FWR should be suspected in patients with moderate pericardial effusion and persistent hypotension especially in the early post MI period. 
2) conservative approach is preferable in those patients with FWR who are hemodynamically stable without evidence of tamponade, probably due to contained rupture.

\section{Patient 2}

A 65 year old hypertensive and diabetic female presented with complaints of persistent chest discomfort since 10 days. At admission her BP was $60 / 30$ mm Hg ECG was suggestive of evolved anterior wall $\mathrm{MI}$ and 2D echo showed hypokinesia in LAD territory, moderate LV dysfunction and large pericardial effusion. Immediate pericardiocentesis was performed however over the next few days she had repeated pericardial collections and her hematocrit had a declining trend and hematocrit of pericardium was similar. Immediate CAG was performed and patient was then transferred to Department of Cardiac Surgery, and exploratory pericardiotomy was performed. During the operation, no definite crevasse was found, but persistent blood ooze on the surface of the heart at the site of trans-mural infarct was noticed by the CT surgeon. Post operatively patient expired due to sepsis.

\section{LEARNING POINT}

Trans-mural MI with hemorrhagic pericardial effusion is one of the frequent causes of post MI pericardial effusion is difficult to from FWR.

\section{Patient 3}

45 year old hypertensive male presented with recent lateral wall MI with persistent post MI angina, however his vitals were stable.2D echo showed moderate pericardial effusion with organized clot in the pericardium which is a contained rupture. Immediate pericardiocentesis was performed. Patient was stabilized and CAG was performed which showed thrombotic lesion in ramus. Patient was discharged in a stable state with advice to continue LMWH and to plan for PTCA after 6 weeks.

\section{LEARNING POINT}

Hyper-echogenic structure in the pericardium in post MI period usually is a contained rupture with pseudo aneurysm.

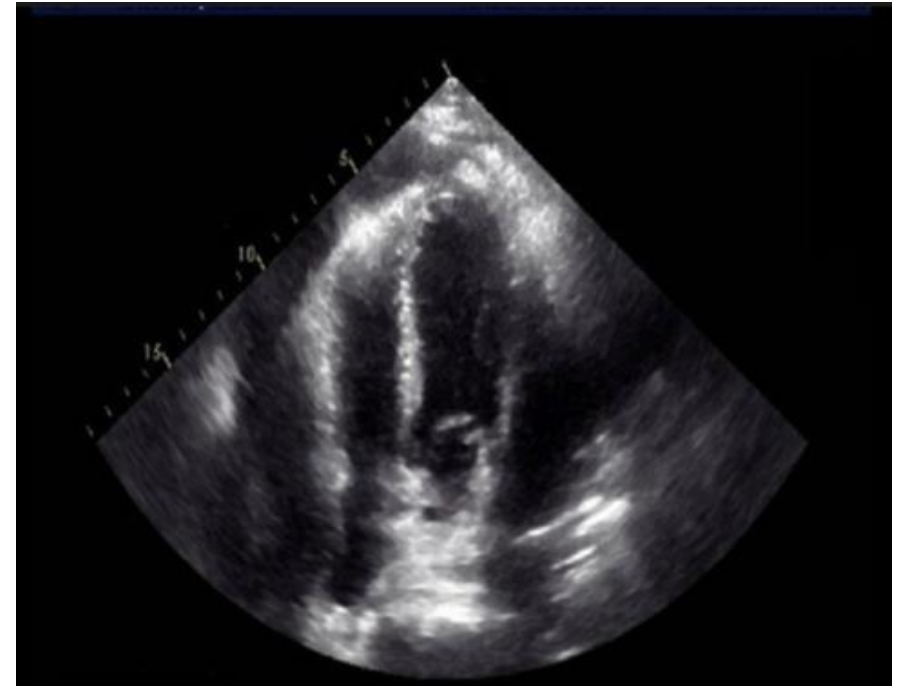

Patient 4

A 50 year old non-hypertensive and non-diabetic male underwent primary PTCA to LAD for AWMI in an outside hospital and discharged in a stable state. He presented with chest pain to EMD 2 days later.

At admission his ECG showed persistent ST segment elevation in all precordial leads similar to previous ECG and 2D echo showed hypokinesia of LAD territory, moderate LV dysfunction and large pericardial effusion without tamponade.

Our initial diagnosis was acute stent thrombosis urgent CAG was performed which revealed a patent stent. Subsequently FWR was considered and cardiac CT was done which failed to show any active leak, probably a contained rupture. Next day he had a cardiac arrest and couldn't be revived.

\section{LEARNING POINT}

Free wall rupture should be suspected in any patient with persistent ST segment elevations and chest pain even after revascularization.

\section{DISCUSSION}

Our experience indicates that STEMI patients with an MPE have increased mortality than those with small PE or without PE, and death was attributable to FWR. Thus, incomplete recanalization of infarct-related coronary artery appears to be a pathophysiological link to hemorrhagic $P E$ and threatening rupture that may cause PE [5]. In addition, the use of temporary pacemakers is 
possibly higher in an interventional population, which also may be associated with right ventricular free wall perforation that may be unnoticed during the procedure. Although detection of a moderate PE early after MI should raise the concern of myocardial rupture, other differential diagnoses should be considered in the acute setting, including aortic dissection and pericarditis $[6,7]$. Angiography and absence of typical rise and fall in cardiac markers often suggest the diagnosis of pericarditis. However, benign early pericarditis after MI may be difficult to differentiate, and development of early pericarditis did not affect long-term outcome [8].

The study by Figueras et al suggests that clinicians should look for PE early, and if moderate PE is present, they should suspect that FWR may be present or evolving. If the PE is hemodynamically compromising, the suspicion of FWR is augmented, especially if electromechanical dissociation occurs. Even in the absence of electromechanical dissociation or cardiac tamponade, mortality was twice that of patients without moderate PE [5].The cause of death in patients with electromechanical dissociation or tamponade is almost exclusively left ventricular FWR occurring within 5 days of STEMI. Figueras et al data demonstrated a clear association between poor ST-segment resolution and presence of PE and especially with tamponade or electromechanical dissociation [5].Figueras et al elegantly demonstrated how measurement of hematocrit in pericardial fluid provides important additional insight into the cause of PE. A diagnostic pericardial puncture with aspiration of 5 to $10 \mathrm{~mL}$ of pericardial fluid can usually be done safely with ultrasound guidance. If the hematocrit level is high, rupture should be considered the cause. If measurement of hematocrit in the pericardial fluid is performed days later, a decline in hematocrit seems ascribable to the formation of a blood clot and the dilution of the supernatant fluid.

\section{Causes of MPE in STEMI}

Broadly patients with MPE after MI can be classified as those with cardiac tamponade (CT) and those without cardiac tamponade (CT)

It is generally accepted that bleeding from the infarcted wall is the most frequent cause of MPE in STEMI patients $[9,10,11,12]$. Accordingly, pericardial fluid in our MPE patients was consistently bloody. In the largest series, pericardiocentesis was hemorrhagic in $75 \%$ of patients [9].

\section{MPE with CT}

Unless proved otherwise FWR is the most probable cause of MPE with CT in the setting of MI, other less common causes include aortic dissection and pericarditis. Common clinical features of sub-acute rupture left ventricular free wall after acute ST segment elevation myocardial infarction are: (1) recurrent or persistent chest pain; (2) recurrent or persistent ST segment elevation; (3) hypotension. Integrating these signs into a syndrome can increase the clinician's awareness to the fatal complication

\section{MPE without Initial CT}

These patients showed demographic and admission ECG characteristics similar to those with CT. The magnitude of MPE, however, was smaller. Thus, it may be that their MPE was mostly secondary to mechanisms other than a myocardial rupture or, conversely, that bleeding caused by the latter was more self-limited than in patients with MPE and CT. As a result of the reduced number of pericardiocentesis procedures, we can speculate that the origin of their MPE is due to contained free wall rupture or due to myopericarditis.

\section{MANAGEMENT AND OUTCOME}

Patients with MPE with CT have a high mortality, and only a few survive with surgical or medical management. Hence, attempts to significantly reduce mortality clearly require a highly motivated and coordinated medical and surgical team eager to initiate resuscitative maneuvers at any time and to rush the patient to the operating room because these are mostly cases of FWR [13, 14].

Patients with MPE without CT, generally considered cases of sub-acute FWR $[9,10,12,15,16]$ manifest lower mortality. A substantial number of them have prompt hemodynamic recovery with pericardiocentesis, and in contrast to other reports $[9,12,13,17-19]$ they can be initially managed medically

Nevertheless, surgery is indicated in those patients who remained hypotensive or have recurrence of CT, although it is not always performed for a number of reasons (comorbidity, persistence of shock, etc.). Despite this approach, 30-day mortality with conservative management is not higher than in operated patients, in existing series $[9,12,19,20]$. 
Patients with MPE without initial CT show a higher mortality than those with small $\mathrm{PE}$ and $8 \%$ die suddenly of a likely FWR (15) The incidence of CT is higher for STEMI patients $(0.9 \%$ to $2 \%)$ compared to other ACS subsets [21, 15].

Unfortunately, no specific features allow identification of those patients who finally developed CT other than when it was a late event. Thus, more refinement is needed in the diagnostic tools aimed to clarify the source of PE and to identify those patients at high risk of EMD. Preliminary observations would suggest that magnetic resonance imaging may help recognize impending FWR [22].

\section{CONCLUSION}

Early diagnosis of etiology of moderate pericardial effusion is important due to its variable clinical course. The most important aspect in the diagnosis of FWR, is to entertain the possibility in the presence of MPE. Hospital prognosis of moderate to severe pericardial effusion (MPE; $10 \mathrm{~mm}$ ) in ST-elevation myocardial infarction is largely unknown, and its management poses a therapeutic dilemma because not all patients benefit from emergency surgery aimed at treating an underlying free-wall rupture.MPE carries an increased mortality, which is highest in patients with CT EMD. Mortality is considerably low after pericardiocentesis, and subsequent management may be individualized because a conservative approach is often successful. Cardiac MRI may aid in identifying the etiology and those at high risk for impending rupture.

It is not only size of effusion but also hemodynamic status, cause is important in the management of post MI pericardial effusion. Acute FWR needs immediate surgery. Sub-acute FWR needs judicious approach with initial medical stabilization followed by surgery.

Hence all patients with post MI MPE should be thoroughly evaluated and managed accordingly.

\section{REFERENCES}

1. Galve E, Garcia-del-Castillo H, Evangelista A, Battle J, Permanyer-Mirarda G, Soler-Soler J. Pericardial effusion in the course of myocardial infarction: incidence, natural history, and clinical relevance. Circulation. 1986;73: 294-299.
2. Guereta P, Khalifeb K, Jobicc Y, Fillipid E et al ; on behalf of the Study Investigators. Echocardiographic assessment of the incidence of mechanical complications during the early phase of myocardial infarction in the reperfusion era: a French multicentre prospective registry. Arch Cardiovasc Dis. 2008;101:41- 47.

3. Lopez-Sendo'n J, Gurfinkel EP, Lopez de Sa E et al; Global Registry of Acute Coronary Events (GRACE) Investigators. Factors related to heart rupture in acute coronary syndromes in the Global Registry of Acute Coronary Events. Eur Heart J. 2010;31:1449 1456.

4. Torp Pedersen C, Hansen FS, Pedersen A. Relation of left ventricular free wall rupture in acute myocardial infarction to forced immobilization. Am J Cardiol. 1988;61:910-912.

5. Figueras J, Barrabe's JA, Serra V, Cortadellas J, Lidón R-M, Carrizo A, Garcia-Dorado D. Hospital outcome of moderate to severe pericardial effusion complicating ST-elevation acute myocardial infarction. Circulation. 2010;122:1902-1909.

6. Sejersten M, Valeur N, Grande P, Toftegaard Nielsen $\mathrm{T}$, Clemmesen P. Long term prognostic value of ST segment resolution in patients treated with fibrinolysis or primary percutaneous coronary intervention. J Am CollCardiol. 2009;54:1763-1769.

7. Figueras J, Juncal A, Carballo J, Cortadellas J, SolerSoler J. Nature and progression of pericardial effusion in patients with a first myocardial infarction: relationship to age and free wall rupture. Am Heart J. 2002; 144:251-258.

8. Imazio M, Negro A, Belli R, Beqaraj F, Forno D, Giammaria M, Trinchero R, Adler Y, Spodick D. Frequency and prognostic significance of pericarditis following acute myocardial infarction treated by primary percutaneous coronary intervention. Am J Cardiol. 2009;103:1525-1529.

9. Lopez-Sendon J, Gonzalez A, Lopez de Sa E, ComaCanella I, Roldan I, Dominguez F, Maqueda I, Jadraque LM. Diagnosis of subacuteventric- ular wall rupture after acute myocardial infarction: 
sensitivity and spec- ificity of clinical, hemodynamic and echocardiographic criteria. J Am Coll Cardiol. 1992;19:1145-1153.

10. Rourke MF. Sub acute heart rupture following myocardial infarction: clinical features of a correctable condition. Lancet. 1973; 2:124 -126.

11. Figueras J, Juncal A, Carballo J, Cortadellas J, SolerSoler J. Nature and progression of pericardial effusion in patients with a first myocardial infarction, relationship with age and free wall rupture. Am Heart J. 2002;144:251-258.

12. Aarseth S, Lange HF. The influence of anticoagulant therapy on the occurrence of cardiac rupture and hemopericardium following heart infarction, I: a study of 89 cases of hemopericardium (81 of them cardiac ruptures). Am Heart J. 1958;56:250-256.

13. Purcaro A, Constantini C, Ciampani N, Mozzanti M, Silenzi C, Gili A, Belardinelli R, Astolfi D. Diagnostic criteria and management of sub acute ventricular free wall rupture complicating acute myocardial infarction. Am J Cardiol. 1997;80:397-405. 18. McMullan MH, Maples MD, Kilgore TL, Hindman $\mathrm{SH}$. Surgical expe- rience with left ventricular free wall rupture. Ann Thorac Surg. 2001;71: 1894 -1898.

14. Shapira I, Isakow A, Burke M, Almog C. Cardiac rupture in patients with acute myocardial infarction. Chest. 1987;92:219-223.

15. Becker RC, Hochman JS, Cannon CP, Spencer FA, Ball SP, Rixzzo MJ, Antman EM. Fatal cardiac rupture among patients treated with thrombolytic agents and adjunctive thrombin antagonists: observations from the Thrombolysis and Thrombin Inhibition in Myocardial Infarction study. J Am CollCardiol. 1999;33:479-487.

16. O Rourke MF. Sub acute heart rupture following myocardial infarction: clinical features of a correctable condition. Lancet. 1973; 2:124 -126.

17. Nunẽz L, de la Llana R, Lopez Sendón J, Coma I, Gil Aguado M, Larrea JL. Diagnosis and treatment of subacute free wall ventricular rupture after infarction. Ann Thorac Surg. 1983;35:525-529.

18. Padro' JM, Mesa JM, Silvestre J, Larrea JL, Caralps J, Cerron F, Aris A. Subacute cardiac rupture: repair with a sutureless technique. Ann Thorac Surg. 1993;55:20-24.

19. Canovas SJ, Lim E, Dalmau MJ, Bueno M, Buendia J, Hornero F, Gil O, Garcia R, Paya R, Perez J, Echanove I, Montero J. Midterm clinical and echocardiographic results with patch glue repair of left ventricular free wall rupture. Circulation. 2003;108(suppl II) II-237-II-240.

20. Bodí V, Monmeneu JV, Marin F. Acute cardiac rupture complicating pre-discharge exercise testing: a case report with complete echocardio- graphic follow-up. Int J Cardiol. 1999;68:333-335.

21. Randomized trial of intravenous streptokinase, oral aspirin, both, or neither among 17,187 cases of suspected acute myocardial infarction: ISIS-2: ISIS-2 (Second International Study of Infarct Survival) Collabo- rative Group. Lancet. 1988;2:349-360.

22. Zoni A, Arisi A, Corradi D, Ardissino D. Magnetic resonance imaging of impending left ventricular rupture after acute myocardial infarction. Circulation. 2003;108:1498-1499. 